\title{
La influencia de Robert Burns en Coleridge y Wordsworth: textos traducidos
}

\author{
C. Huertas Abril/J. M. Castellano Martínez \\ (G.I. Lexicografía y Traducción Literaria, HUM-198)
}

Fecha de recepción: 12 de febrero de 2009

Fecha de aceptación: 15 de abril de 2009

Resumen: El presente trabajo se ocupa de la figura del poeta escocés Robert Burns, que influencia a los poetas 'lakistas', especialmente a Coleridge y a Wordsworth. Tras una breve referencia a la vida y a la obra de Burns, se analiza el patriotismo en Reino Unido a finales del siglo XVIII. Finalmente, se traducen al español tres poemas, "Lament of Mary Queen of the Scots On the Approach of Spring" de Burns, "To the Exiled Patriots" de Coleridge y "When I have borne in memory" de Wordsworth, que ponen de manifiesto la relación entre los tres autores.

Palabras clave: Robert Burns, Coleridge, Wordsworth, Traducción poética

Abstract: This paper aims to study the Scottish poet Robert Burns, who is a noticeable influence for the Lake poets, especially for Coleridge and Wordsworth. After a brief reference to the biography and works written by Burns, we analyse the nationalistic movement in the United Kingdom during the second part of the $18^{\text {th }}$ century. Finally, we translate into Spanish three poems "Lament of Mary Queen of the Scots On the Approach of Spring" by Burns, "To the Exiled Patriots" by Coleridge y "When I have borne in memory" by Wordsworth, which show the existing influence between these authors.

Key words: Robert Burns, Coleridge, Wordsworth, Poetic Translation

\begin{abstract}
INTRODUCCIÓN
Al igual que en la mayoría de los países europeos, desde la segunda mitad del siglo XVIII surge en el Reino Unido un grupo de poetas que por sus obras ya manifiestan ideas y tendencias más propias del Romanticismo que de su época. Por ello, si tuviéramos que fechar el comienzo de la literatura romántica en Gran Bretaña, ese año sería 1798, cuando se publica la primera edición de las Lyrical Ballads. Los autores de esta obra, Samuel Taylor Coleridge y William Wordsworth, son conocidos, junto con Robert Southey, como los poetas 'lakistas'. La temática que abarca la poesía lakista es muy variada: naturaleza, mitos, leyendas, ideales políticos y sentimientos de nostalgia. Dos años antes de la publicación de la obra anterior, fallece uno de los escritores escoceses más universales, Robert Burns, el "poeta nacional" de Escocia, cuyas obras son leídas por los 'lakistas'.
\end{abstract}


Tanto Robert Burns como los poetas 'lakistas', provenientes de Escocia e Inglaterra respectivamente, viven en un período histórico en el que la identidad de Gran Bretaña se define, más por necesidad que por voluntad, como nación. Por una parte, Burns exaltará en sus poemas la identidad de Escocia frente a Inglaterra, como respuesta a la impopularidad de la Act of Union de 1707 mediante la que el Reino Unido de la Gran Bretaña se constituye como país; por otra, los poetas 'lakistas' beberán del sentimiento de este poeta y manifestarán sus ideas nacionales, en un principio a favor de los principios de la Revolución Francesa y más tarde en contra de los mismos, debido, entre otros factores, a los constantes intentos de invasión de las islas británicas por parte de Napoleón Bonaparte.

Este trabajo pretende analizar la influencia que Robert Burns tiene en la obra de dos poetas 'lakistas', Coleridge y Wordsworth, y centra su atención en la temática del patriotismo y el sentir nacional. Una vez se estudie la vida y contexto histórico de dichos autores, proponemos una versión traducida al español de tres poemas relacionados con esta temática.

\section{ROBERT BuRns: "THE PloughmAN POET"1}

Robert Burns, considerado el pionero del Romanticismo en la Europa dieciochesca, es conocido no sólo por ser el poeta más importante en lengua escocesa ${ }^{2}$, sino también por ser fuente de inspiración para los fundadores del liberalismo y los promotores de los derechos sociales y la igualdad entre hombres y mujeres. Este "Ploughman poet", figura que tanto anhelaba la sociedad escocesa de su época, promueve el sentimiento nacional, que los poetas 'lakistas' extrapolarán a la exaltación y defensa del Reino Unido ante las intervenciones militares de Napoleón en Europa. Burns es consciente de que escribir en escocés limitaría la recepción de su obra. Por este motivo, en sus poemas combina de forma sutil la lengua escocesa con la inglesa.

Nacido en el sur de Ayr, en South Ayrshire, en el año 1759, Robert Burness $^{3}$ es el mayor de siete hermanos de una familia humilde, lo que condicionará su obra. Su padre, a pesar de numerosos intentos para sacar a su familia de la pobreza y de las dificultades que tienen que afrontar, no

\footnotetext{
${ }^{1}$ Uno de los apodos con los que se conoce a Robert Burns y que podría traducirse como el "poeta labrador", haciendo alusión a sus orígenes humildes y a su sencillez en el trato con los agricultores de su región. Asimismo, Burns es conocido con otros sobrenombres, entre los que destacan "Scotland's favourite son", el "Bard of Ayrshire" o simplemente "the Bard", o el diminutivo "Rabbie" Burns.

${ }^{2}$ En este y en los casos sucesivos hablamos de lengua escocesa y no de dialecto debido a que la Carta Europea de las Lenguas Minoritarias o Regionales la reconoce como tal desde 1992. Existen, con todo, al menos cinco dialectos diferentes dependiendo de la región en que se utilice.

${ }^{3}$ Decidirá actualizar su apellido en 1786 y cambiarlo por Burns.
} 
impide que Robert comience a ir a la escuela a los seis años. Allí aprende rápidamente a leer y a escribir, desarrollando una afición por los libros poco común a su edad. Asimismo, aprende gramática inglesa, lo que posteriormente le permitirá utilizar el inglés junto con su lengua materna, el escocés. No obstante, Robert ha de ayudar en las distintas tareas de la granja, un negocio que a la familia no sólo no le reportará beneficios, sino que los llevará a la ruina.

Con 15 años, Robert Burns afirma que, por primera vez, "commited the sin of rhyme" [James Currie, 1826: iii], pues comienza a escribir versos a las jóvenes que, como él, trabajan en los campos, siendo su primer poema "O, Once I Lov'd A Bonnie Lass". Además, sigue manteniendo su amor por la lectura, y será entonces cuando lea obras de Shakespeare, Pope, Locke y Taylor, entre otros. Tras la muerte de su padre, Burns comienza a destacar en la región donde vive, gracias a su cultura y a sus versos, pues tras un período sin dedicarse a ellos, retoma la composición de poemas dedicados a mujeres, prefiriendo siempre a aquéllas de orígenes más humildes.

A pesar de sus numerosos escarceos amorosos y su deseo de viajar a Jamaica, Burns logra en su tierra natal el reconocimiento hacia sus poemas, que atraen a todo tipo de público. Así, a sugerencia de uno de sus hermanos, publica Poems, Chiefly in the Scottish dialect, conocido habitualmente como "the Kilmarnock volume"4. El éxito de la obra es inmediato, y se le comienza a conocer en todo el país. Por este motivo, es invitado a Edimburgo para publicar una segunda edición, de dos mil ejemplares, donde es recibido por todas las personalidades de la capital. Como señala Currie (1826: iv):

But what contributed principally to extend his fame into the sister kingdom, was his fortunate introduction to Mr. Mackenzie, who, in the $97^{\text {th }}$ paper of the Lounger, recommended his poems by judicious specimens, and generous and elegant criticism. From this time, whether present or absent, Burns and his genius were the objects which engrossed all attention and all conversation.

Comienza a asistir a distintos clubes de la élite de Edimburgo, donde sorprende a sus iguales con ingeniosos juegos de palabras, mientras se muestra sumamente decoroso con aquéllos de rango superior al suyo. Traba grandes amistades con Lord Glencairn y con Frances Anna Dunlop,

${ }^{4}$ Editado por John Wilson, la primera edición tiene una tirada de 600 ejemplares. Este volumen contiene, además de diversas sátiras, poemas como "The Twa Dogs" y "The Cotter's Saturday Night", excelentes descripciones de la vida campesina escocesa. 
quienes lo apoyan en numerosas ocasiones. Se enamora de Agnes "Nancy" McLehose, con quien intercambia una amplia correspondencia durante varios años ${ }^{5}$ y que concluye cuando, en 1791, ella parte a Jamaica, justo antes de lo cual Burns le remite su poema "Ae Fond Kiss". Asimismo, Burns simpatiza con la Revolución Francesa hasta que se desarrollan las guerras franco-británicas, en las que por motivos patrióticos participa en una expedición contra los franceses.

Sus distintos viajes a Edimburgo comienzan a mostrarle que no llegará nunca a poder instalarse allí, por lo que finalmente se instala en una granja cercana a Dumfries para poder tener una familia. Con todo, este cambio en su vida dará origen a una gran infelicidad que predominará en su futuro. Tras un período de sosiego, lejos de la capital, retoma de nuevo su afición por los clubes sociales; Carlyle (1838:329) describe la actitud de Burns:

His conversation expressed perfect self-confidence, without the slightest presumption. Among the men who were the most learned of their time and country, he expressed himself with perfect firmness, but without the least intrusive forwardness ; and when he differed in opinion, he did not hesitate to express it firmly, yet at the same time with modesty. [...] I remember, on this occasion I mention, I thought Burns'a acquaintance with English poetry was rather limited; and also, that having twenty times the abilities of Allan Ramsay and of Fergusson, he talked of them with too much humility as his models: there was doubtless national predilection in his estimate.

En 1792 le solicitan su colaboración en la Thomson's Collection of Scottish Songs ${ }^{6}$, para lo que escribe sin pausa alguna aproximadamente seiscientas canciones, que se distribuirán en los tres volúmenes de la colección. Por otra parte, su gusto por los libros lo lleva a distribuir algunos

\footnotetext{
${ }^{5}$ En las cartas ambos utilizan pseudónimos: mientras que Burns es "Sylvander", McLehose tiene el sobrenombre de Clarinda.

${ }^{6}$ Su promotor, George Thomson (1757-1851), es editor de canciones populares escocesas. Desarrolla su interés por el folklore escocés en la última década del siglo XVIII y decide publicar una colección para voz y piano, por parte de los compositores europeos más destacados. En el prefacio de "Select Collection of Scottish Airs" afirma: "The Symphonies and Accompaniments next engaged his solicitude. For the composition of these, he entered into terms with Mr Pleyel, who fulfilled part of his engagement satisfactorily; but having then stopt short, the Editor found it necessary to turn his eyes elsewhere. He was so fortunate, however, as to engage Mr Kozeluch, and afterwards Dr Haydn, to proceed with the Work, which they have finished in such a manner as to leave him nothing to regret on Mr Pleyel's breach of engagement."
} 
ejemplares entre sus vecinos, descuidando así su granja y perdiendo la producción.

La producción literaria de Burns consiste, a partir de entonces, casi enteramente en canciones populares. En Dumfries compone más de cien nuevas canciones para The Melodies of Scotland, y colabora también con $A$ Select Collection of Original Scottish Airs for the Voice, y muchos de sus poemas más conocidos, como "Auld Lang Syne" o "A Red, Red Rose" se encuentran ahí recogidos. La mañana del 21 de julio de 1796, Robert Burns fallece en la pobreza, aunque no arruinado, en Dumfries con 37 años.

\section{EL PATRIOTISMO EN REINO UNIDO A FINALES DEL SIGLO XVIII}

\subsection{Antecedentes y repercusiones: Act of Union (1707)}

Los primeros intentos de unión entre los reinos de Inglaterra y Escocia se remontan a finales del siglo XIII y principios del XIV. Sin embargo, dicha unión no se hará efectiva hasta 1707, año en el que los parlamentos nacionales de ambos países aprueban la "Act of Union", por la que surge el Reino Unido de Gran Bretaña.

Los motivos que impulsan la forja de una "identidad" británica entre ambas naciones encuentran su origen en intereses diversos. Por una parte, Inglaterra aseguraría la continuidad en la corona de la fe protestante en ambos reinos ${ }^{8}$ para evitar de este modo que Escocia eligiera a un monarca que profesara el catolicismo; por otra, esta última obtendría beneficios fiscales y financieros de las colonias inglesas de ultramar, desde entonces británicas, que permitirían sanear las arcas de la economía escocesa tan debilitada por el "Darien scheme", los aranceles comerciales continentales y la hegemonía naval y marítima de Inglaterra.

Finalmente, el 16 de enero de 1707 se aprueba la "Act of Union", que entra en vigor el 1 de mayo de ese mismo año y que disuelve el parlamento escocés para unir a los reinos de Escocia e Inglaterra en un mismo país. No obstante, Escocia conserva su independencia en lo que concierne a la administración judicial y religiosa, mientras que comparte con Inglaterra comercio, soberanía, impuestos y parlamento (reunido por primera vez en el

\footnotetext{
${ }^{7}$ En este período tienen lugar las guerras de independencia de Escocia. Los intentos invasores del reino de Inglaterra derivaron siempre en largas guerras. El Tratado de EdimburgoNorthamptom puso fin a la primera de las guerras (1296-1328), mientras que la firma del Tratado de Berwick trajo la paz a ambas naciones tras la segunda guerra (1332-1357).

${ }^{8}$ En 1603 , el rey de Escocia, Jacobo VI de Estuardo, accede al trono de Inglaterra tras la muerte de la última Tudor, Isabel I, quien muere sin descendencia. Desde entonces, ambos reinos comparten monarca aunque con parlamentos y legislaciones independientes.

${ }^{9} \mathrm{~A}$ finales del siglo XVII, en un intento de expansión colonial, el reino de Escocia pretende establecer una colonia en el actual istmo del Panamá a la que daría el nombre de "Nueva Caledonia". Este fracasado proyecto quiso hacer frente a la difícil situación económica en la que se sumergía Escocia debido a la debilidad de su posición política y geográfica en Europa.
} 
mes de octubre de dicho año). Otra de las insignias que comparten ambos reinos desde entonces es la bandera, que combina la cruz roja de San Jorge con la cruz azul de San Andrés, popularmente conocida como "Union Jack"10. Por el contrario, la complejidad de este "tratado" se pone de manifiesto en las discrepancias políticas existentes entre los pueblos escocés e inglés. En Escocia existe una sociedad resentida por la pérdida de soberanía nacional con la desaparición del parlamento escocés que ya desde 1689 había comenzado a perder atribuciones por parte del monarca de Londres. La opinión pública imperante en la Escocia de aquella época queda reflejada en el famoso poema de Robert Burns, "A parcel of rogues in a nation"11, de los que destacamos los siguientes versos (Cunningham, 1834: 399):

(...) a coward few,

For hireling traitors' wages...

We're bought and sold for English gold-

Such a parcal of rogues in a nation!

\subsection{Relaciones franco-británicas en el contexto de la Revolución Francesa}

El estallido de la Revolución Francesa a finales del siglo XVIII supone un antes y un después en la Historia (Cody, 1987). Los ideales antimonárquicos que surgen en Francia a partir de 1789 mantienen en vilo a todos los reinos de Europa. Mientras el país galo se sumía en el caos, Gran Bretaña aprovecha para desarrollar su poderío naval.

En un principio, los intelectuales británicos ven con buenos ojos los ideales revolucionarios ya que, además de debilitar la presencia política de Francia en Europa, afianza los valores liberales de la monarquía parlamentaria del Reino Unido (Philp, 2002). Son varias las obras consagradas o bien inspiradas en la Revolución Francesa por parte de los poetas 'lakistas': France: An Ode, de Coleridge (1798) o The Prelude, de Wordsworth (1805); así como el poema Tree of Liberty, de Robert Burns (1793). Este movimiento político-social repercute, pues, en la literatura inglesa y sus autores (O. Grenby, 2008):

II est largement admis que la Révolution française a eu un impact énorme sur la littérature anglaise et que pratiquement tous les poètes, romanciers et auteurs dramatiques de cette époque ont écrit des

\footnotetext{
${ }^{10}$ Una nueva versión de esta bandera surge tras la Act of Union de 1800, año en el que la cruz roja de San Patricio se suma a la Union Jack con motivo de la anexión de Irlanda al Reino Unido.

${ }^{11}$ Las palabras del escritor escocés reflejan el sentir nacional de un pueblo al que la élite junto con la aristocracia de la "parcel of rogues", es decir, el parlamento, habían "comprado y vendido por oro inglés"
} 
textes influencés par les principes et par les remarquables événements français.

Sin embargo, la fascinación por los valores liberales franceses pronto desaparecerá de los círculos intelectuales. Edmund Burke ${ }^{12}$, en su obra Reflections on the Revolution in France (1790), critica los valores revolucionarios franceses, ya que, según él:

It appears to me as if I were in a great crisis, not of the affairs of France alone, but of all Europe, perhaps of more than Europe. All circumstances taken together, the French revolution is the most astonishing that has hitherto happened in the world. The most wonderful things are brought about, in many instances by means the most absurd and ridiculous, in the most ridiculous modes, and apparently by the most contemptible instruments. Everything seems out of nature in this strange chaos of levity and ferocity, and of all sorts of crimes jumbled together with all sorts of follies. In viewing this monstrous tragicomic scene, the most opposite passions necessarily succeed and sometimes mix with each other in the mind: alternate contempt and indignation, alternate laughter and tears, alternate scorn and horror.

Tras los intentos fallidos de instaurar una monarquía constitucional en Francia, Luis XVI es sometido a juicio en 1792 y ejecutado un año más tarde. Ante tal situación, Reino Unido forma una coalición junto a otras naciones europeas, como España y Portugal, y declara la guerra a la Revolución. En 1798 el ejército francés envía una flota a Irlanda para prestar auxilio a la "Society of United Irishmen"13, organización que pretende liberar a Irlanda de la dominación británica para crear "otra" República. El temor a que las pretensiones irlandesas se cumplan impulsa la firma de una nueva "Act of Union" en 1800, mediante la cual Irlanda se integra en la corona del Reino Unido a partir del año siguiente.

\subsection{Desencanto por los valores franceses: el Imperio Napoleónico}

En un principio, los valores franceses que surgen en la Revolución de 1789 inspiran a gran parte de los escritores y eruditos británicos de

\footnotetext{
${ }^{12}$ Escritor y crítico británico nacido en Dublín de ideología liberal-conservadora contrario a la Revolución Francesa. Además de Reflection on the Revolution in France (1790), es también autor de A Philosophical Enquiry into the Origin of Our ldeas of the Sublime and Beautiful (1757).

13 Organización de política liberal fundada en el siglo XVIII inspirada en los ideales de la revolución de las Trece Colonias así como en los valores de la Revolución Francesa. Los principales objetivos de esta organización se centraban en la independencia de Irlanda respecto a Gran Bretaña.
} 
pensamiento liberal y progresista, como los poetas 'lakistas'. Sin embargo, los acontecimientos que se desarrollan tras el nombramiento de Napoleón Bonaparte como primer cónsul de Francia, distorsionan las ideas de estos intelectuales.

En 1803, Napoleón invade Hannover, posesión británica, y un año después concentra tropas y una flota en Boulogne-sur-Mer, con vistas a un desembarco. Sin embargo, en octubre de 1805, la victoria británica de Trafalgar acaba con las pretensiones invasoras de Napoleón. El gobierno británico intenta llegar a un acuerdo con la Francia napoleónica pero fracasa en su intento. La respuesta gala consistió en llevar a cabo el famoso bloqueo continental que prohíbe la llegada de suministros a Gran Bretaña. Tras las invasiones napoleónicas por toda Europa, Reino Unido lleva a cabo una serie de batallas navales contra Francia que terminan en victoria ${ }^{14}$, hecho que afianza el dominio británico de los mares.

En la batalla de Trafalgar fallece el Almirante Nelson, uno de los marinos más célebres de la historia naval (Amado, 2009). Horatio Nelson, nacido en la región inglesa de Norfolk, se convierte tras su muerte en leyenda y figura romántica, que reforzaría la identidad británica frente al Imperio napoleónico. Prueba de ello son las numerosas obras consagradas a su persona, como The Life of Horatio Lord Nelson ${ }^{15}(1813)$ del poeta lakista Robert Southey, que pone de manifiesto el sentimiento patriótico:

My mind was staggered with a view of the difficulties I had to surmount and the little interest I possessed. I could discover no means of reaching the object of my ambition. After a long and gloomy reverie, in which I almost wished myself overboard, a sudden glow of patriotism was kindled within me, and presented my king and country as my patron. 'Well then,' I exclaimed, 'I will be a hero! and, confiding in Providence, I will brave every danger!'

La caída del I Imperio francés y enemistad con Napoleón, el desencanto por los valores de la Revolución Francesa antaño admirados, y el auge del poderío económico, industrial y militar del Reino Unido de Gran Bretaña e Irlanda propician el surgimiento del Romanticismo inglés de la mano de los poetas 'lakistas' Coleridge, Wordsworth y Southey (Whitney, 2000).

\footnotetext{
${ }^{14}$ Destacan las victorias británicas en las batallas navales de Copenhague (1801) y Trafalgar (1805).

${ }^{15}$ La película cinematográfica británica Nelson, proyectada en el año 1926, fue una adaptación de esta obra.
} 
3. EL PATRIOTISMO DE ROBERT BURNS Y SU INFLUENCIA EN LOS POETAS 'LAKISTAS'

\subsection{Robert Burns, hijo predilecto de Escocia}

La importancia de Robert Burns se debe tanto a sus obras poéticas y recopilaciones de canciones tradicionales, como a su labor de ensalzamiento de la identidad escocesa. El "bardo de Escocia" ha adquirido dicha denominación por su sofisticado estilo de escritura, que combina las lenguas escocesa e inglesa y que le facilita la expresión de sus sentimientos junto con una voz genuina para el nacionalismo escocés. Especialmente, la "Kilmarnock edition" de Poems, Chiefly in the Scottish Dialect crea un efecto revulsivo para detener las presiones contra los escoceses, que predominan en el siglo XVIII. Las "Union Acts" suponen la imposición de la lengua inglesa, que comienza a asociarse con el poder en la ideología política de Escocia y, por consiguiente, acarrean consecuencias tanto lingüísticas como en la identidad nacional y cultural. El cambio en el estatus de las lenguas conlleva la introducción de un nuevo sistema educativo en el cual comienza a menospreciarse la lengua escocesa. Así, la sociedad trata de adoptar el acento inglés y presenta en las escuelas la lengua inglesa como "remedio" a la vulgaridad escocesa. Paradójicamente, esta situación coincide con la edad de oro de Escocia, cuyos máximos exponentes no dudan en rechazar su lengua materna. Por citar tan sólo un ejemplo, Hume (1983:389), en una de sus cartas, afirma:

Is it not strange that, at a time when we have lost our Princes, our Parliaments, our independent Government [...] are unhappy, in our Accent and Pronunciation, speak a very corrupt Dialect of the Tongue we make use of; is it not strange, I say, that in these Circumstances, we shou'd really be the People most distinguish'd?

Tan solo la poesía en lengua escocesa se considera una excepción aceptable a la idea de vulgaridad, pues se asocia con los sentimientos, si bien alejada de la lengua inglesa, intelectual y racional. En este contexto, Robert Burns utiliza las técnicas de dos de sus más ilustres predecesores, Ramsay y Fergusson, y junto a ellos consigue hacer renacer la lengua vernácula y devolverla al estatus que había perdido. Así, mientras que algunos escritores escoceses, como Blackdock o Blair, escriben en inglés estándar, Burns, al defender el escocés, es consciente de su papel político y, como afirma McClure (1995:30) se trata de "an act with overt and inescapable cultural, even political, implications: a delibérate gesture of support for a denigrated tongue".

Burns publica durante su vida cientos de poemas, canciones populares y cartas. Algunas de sus obras las escribe sólo en escocés, o expresamente en inglés (especialmente cuando tiene a lectores británicos 
como destinatarios o cuando trata temas de carácter político), si bien la mayoría de ellas combinan ambas lenguas y distintas modulaciones en el registro. La crítica suele asociar esta inusual combinación lingüística a la evaluación de la presencia de cuestiones políticas, como "The Twa Dogs" (crítica al calvinismo) o "A Man's A Man For A' That" (concienciación sobre las clases sociales), o de carácter patriótico, como "Scots Wha Haw", "Robert Bruce's March to Bannockburn", "Gude Wallace" o "Lament of Mary, Queen of the Scots, On the Approach of Spring"16.

No obstante, hasta en los poemas en los que se aprecia una menor carga política, el patriotismo de Burns resulta manifiesto, lo cual contribuye a su consideración como hijo predilecto de Escocia, e incluso como símbolo nacional. De hecho, el propio poeta se describe en el prefacio de la segunda edición de Poems, Chiefly in the Scottish Dialect como "A Scottish Bard, proud of the name, and whose highest ambition is to sing in his Country's service". Su implicación política y su compromiso con Escocia es aún más patente en el último poema de dicha obra, "A Bard's Epitaph"17, cuya segunda estrofa reproducimos:

Is there a bard of rustic song,

Who, noteless, steals the crowds among,

That weekly this area throng, $\mathrm{O}$, pass not by!

But, with a frater-feeling strong, Here, heave a sigh.

Al mostrarse como un "ploughman poet" y, literalmente, como un "bard of rustic song", Burns pasa de ser un héroe local a una personalidad nacional que defiende los intereses de sus compatriotas, abogando de forma simultánea por los derechos sociales. La clase alta de Edimburgo lamenta, como señala Low (1974:91) el uso de un "uncouth dialect" (dialecto inculto o incluso, por la ambigüedad del término utilizado, grosero). Con todo, son muchos los que advierten las connotaciones sociolingüísticas en la obra de Burns y, mediante ella, como afirma McGuirk (1958:118) "disseminated Scots vernacular [and his ideology] into the vocabulary of the world".

\footnotetext{
${ }^{16}$ Proponemos una traducción para este poema en el apartado cuarto del presente trabajo, titulado "Textos traducidos".

17 Wordsworth (1855:488) escribe sobre este poema manifestando su admiración por Burns: "Who but himself -himself anticipating the but too probable termination of his own course? Here is a sincere and solemn avowal -a confession at once devout, poetical, and human -a history in the shape of a prophecy!"
} 


\subsection{El patriotismo y la influencia de Burns en Coleridge y Wordsworth}

A lo largo de su juventud, William Wordsworth y Tylor Coleridge sienten una profunda admiración hacia el fervor de los ideales y valores que promueve la Revolución Francesa. Así lo demuestra Coleridge en France: An Ode (1798) o Wordsworth en The Prelude (1805) ${ }^{18}$ :

Twas in truth an hour

Of universal ferment; mildest men

Were agitated; and commotions, strife

Of passion and opinion, filled the walls

Of peaceful houses with unique sounds.

The soil of common life, was, at that time,

Too hot to tread upon. ${ }^{19}$

Pero la irrupción de Napoleón en la escena internacional rompe todos los esquemas liberales de estos poetas ingleses. Woods (2003) afirma al respecto:

But as Bonaparte crushed the most radical elements they became disillusioned and moved back to the right. This is a phenomenon seen many times in history, where the intellectuals and artists (with some notable exceptions) swing to the left and right with the ups and downs of the revolution.

Aunque el sentimiento romántico inglés que se inicia con estos poetas abarque temas tan variopintos como son la naturaleza, la nostalgia, el misterio, el mito o las ruinas; destacan en estos autores una serie de obras y composiciones que reflejan cierto sentir nacional, que se asemejan al de Burns por Escocia. Así, Coleridge colabora junto con Southey en una obra titulada The Fall of Robespierre (1795) y expone años después sus ideales más conservadores sobre los asuntos políticos de la nación en On the Constitution of Church and State (1830). Por su parte, William Wordsworth, de carácter más independiente y solitario, compone poemas relacionados con la grandeza de nación en épocas pasadas, como Lines composed a few

\footnotetext{
${ }^{18}$ Pese a que esta obra fuera compuesta por William Wordsworth en 1805, no fue publicada hasta 1850.

${ }^{19}$ Extracto del noveno libro de The Prelude, titulado Residence in France; en el que William Wordsworth relata en verso las vivencias que tuvo durante su estancia en la Francia revolucionaria.
} 
miles above Tintern Abbey on revisting the Banks of the Wye Valley during tour, July $13,1798^{20}$ o The Prelude.

\subsubsection{Samuel Taylor Coleridge}

Considerado uno de los fundadores del movimiento literario romántico en Inglaterra así como uno de los máximos exponentes de los escritores 'lakistas', Coleridge destaca en el ámbito de la poesía, la crítica y la filosofía. Su poema por excelencia es The Ryme of the Ancyent Marinere, en el que narra la historia de un marinero durante su largo viaje por mar.

Samuel Taylor Coleridge nace el 21 de octubre de 1772 en la localidad inglesa de Ottery St. Mary (Devon), de la que es reverendo su padre, John Coleridge. Tras la muerte de éste, Coleridge queda huérfano e ingresa en el internado Christ's Hospital School de Londres. En febrero de 1791 se traslada al Jesus College, en Cambridge, donde asiste a clases de forma irregular hasta 1794, año en el que abandona la universidad sin obtener título alguno. Ese mismo año visita Oxford y posteriormente Bristol, donde conoce a Robert Southey, quien le trasmite su admiración por los valores de la Revolución Francesa y la teoría de la sociedad de la Pantisocracia. De esta amistad surge una obra dramática, The Fall of Robespierre (1794), de la que escribe el primer acto.

En 1795 contrae matrimonio con Sarah Fricker, de quien nunca llega a enamorarse, y comienza a impartir docencia en el Bristol College. En el verano de ese mismo año conoce a los hermanos William y Dorothy Wordsworth, con quienes mantendrá una gran amistad. Un año más tarde, en 1796, Coleridge publica Poems on Various Subjects, su primera colección de poemas. Posteriormente, William Wordsworth finaliza Lyrical Ballads (1798), obra en la que Coleridge compone varios poemas, de entre los que destaca The Ryme of the Ancyent Marinere. Esta obra supone el punto de partida del Romanticismo literario en Inglaterra. En otoño de ese mismo año inicia un viaje a Alemania junto con los hermanos Wordsworth. A finales de 1799 conoce a Sarah Hutchinson, futura cuñada de su amigo Wordsworth, de quien se enamora y a quien dedica su obra Dejection: An Ode (1802). En la segunda edición de Lyrical Ballads, publicada el mismo año que la obra anterior, Coleridge añade un nuevo poema, Love, dedicado también a Sarah.

En 1816 vieron la luz los poemas inacabados de Christabel y Kubla Khan, inspirado en un sueño premonitorio según el propio poeta. Un año más tarde, en 1817, publica Sibylline Leaves y Biographia Literaria, su obra más importante en este período. En 1824 ingresa como miembro electo en

${ }^{20}$ En este poema se describe la abadía de Tintern, en el país de Gales. Wordsworth busca las raíces históricas de este lugar y las relaciona con el paraje natural que le rodea. Este poema forma parte del volumen I de las Lyrical Ballads. 
la Royal Society of Literature ${ }^{21}$. Finalmente, Coleridge fallece el 25 de julio de 1834 en Highgate, a las afueras de Londres.

3.2.2. William Wordsworth

Uno de los más importantes poetas románticos de lengua inglesa y poeta lakista por antonomasia, William Wordsworth es autor, junto con su amigo Coleridge, de las Lyrical Ballads, colección de poemas con la que se inicia el movimiento romántico en Inglaterra.

Hijo de John Wordswrth y Ann Cookson nace el 7 de abril de 1770 en Cockermouth (Cumberland). Un año más tarde nace su hermana Dorothy, con quien mantiene una estrecha relación a lo largo de toda su vida. Al quedar huérfano de madre en 1778, ingresa en el Hawkshead Grammar School. En 1787 debuta como escritor al publicar un soneto en The European Magazine y asiste al St. John's College, en Cambridge, donde obtendrá el título de Bachelor of Arts. En 1790 inicia un viaje por Europa visitando los Alpes y distintas regiones de Suiza, Italia y Francia. En 1791 se dirige a éste último país, que se encuentra en plena revolución, y se impregna de los ideales del movimiento republicano. Allí se enamora de Annette Vallon en 1792, con quien tiene a su primera hija, Caroline; sin embargo, las complejas relaciones diplomáticas entre Gran Bretaña y Francia suponen su retorno a Inglaterra. Finalmente, en 1802 ambas naciones firman la Paz de Amiens, lo que le posibilita la vuelta a Francia de Wordsworth para visitar a Annette y Caroline.

En 1798 publica, en colaboración con Coleridge, la obra clave del Romanticismo inglés: Lyrical Ballads. La mayoría de los poemas de la primera edición pertenecen a Wordsworth, siendo tan sólo cuatro las aportaciones de Coleridge (entre las que se encuentra The Ryme of the Ancyent Marinere). Posteriormente se realiza una segunda edición en 1800 y una reedición de ésta en 1802. En otoño del mismo año en el que se publica la primera edición de Lyrical Ballads inicia, acompañado por Dorothy y Coleridge, un viaje a Alemania, donde compone The Prelude así como varios de los poemas que se incluyen en The Lucy Poems. Más tarde regresa a Inglaterra con su hermana y se instalan en el Dove Cottage, en Grasmere, localidad del Lake District. En esta época conoce a Southey, de manera que con Coleridge se establece así la amistad entre los tres grandes poetas 'lakistas'.

En 1803 contrae matrimonio con Mary Hutchinson, con quien tiene cinco hijos. Cuatro años después publica Poems in Two Volumes y en 1814 The Excursion. En 1830 la Universidad de Durham otorga el título de Doctor of Civil Law, al igual que la Universidad de Oxford hace un año más tarde.

${ }^{21}$ Organización literaria británica fundada en 1820 por el rey Jorge IV. La principal finalidad de esta sociedad consiste en el reconocimiento y mérito literario. El primer presidente de la Sociedad es Thomas Burgess, posteriormente obispo de Salisbury. 
El 23 de abril de 1850 fallece, siendo sus restos incinerados en la iglesia de St. Oswald (Grasmere). 


\title{
4. TEXTOS TRADUCIDOS
}

\section{1. "Lament of Mary, Queen of the Scots, On the Approach of Spring", de Burns}

LAMENT OF MARY, QUEEN OF SCOTS, ON THE APPROACH OF SPRING ${ }^{22}$

Now Nature hangs her mantle green

On every blooming tree,

And spreads her sheets o' daisies white

Out o'er the grassy lea;

Now Phoebus cheers the crystal streams,

And glads the azure skies;

But nought can glad the weary wight

That fast in durance lies.

Now laverocks wake the merry morn

Aloft on dewy wing;

The merle, in his noontide bow'r,

Makes woodland echoes ring;

The mavis wild wi' mony a note,

Sings drowsy day to rest:

In love and freedom they rejoice,

Wi' care nor thrall opprest.

\begin{abstract}
22 Burns, con el presente poema escrito en 1791, pretende rendir tributo a María Estuardo, conocida en Escocia como Mary, "Queen of the Scots", y poner así de manifiesto su patriotismo. María Estuardo, nacida en 1542, tras la muerte de su padre de forma prematura, es coronada como reina de Escocia con apenas nueve meses. Con cinco años la prometen en matrimonio con Eduardo, hijo de Enrique VIII de Inglaterra, mediante los tratados de Greenwich. Sin embargo, debido a la oposición del pueblo escocés y de las exigencias de Inglaterra, esta unión se rompe desencadenándose numerosas batallas en Escocia, y María es enviada a Francia, donde pasa trece años en la Corte y contrae matrimonio con Francisco II, convirtiéndose así en reina de Escocia y de Francia. En 1560 queda viuda y decide regresar a su tierra natal, casándose cinco años después de forma inesperada con Enrique Estuardo, uno de los principales líderes católicos. Esto desencadena una revolución abierta por parte de los protestantes, que la lleva a refugiarse en Stirling. Su matrimonio también tiene consecuencias en Inglaterra, pues al haberse casado con un noble inglés, la posible descendencia podría tener derechos para la sucesión en el trono, como así será con Jacobo. En 1567 es secuestrada y obligada a abdicar en su hijo, de apenas un año de edad. Sin embargo, logra escapar pero en su huída a Inglaterra es capturada y sometida a juicio por la muerte de su segundo marido. Es entonces cuando hace su famosa afirmación "En ma fin gît mon commencement". Muere decapitada en 1587, aunque su figura sigue siendo una de las más importantes para la sociedad escocesa.
\end{abstract}


LAmento de María, Reina de los Escoceses, en LA LLEGAdA de LA PRIMAVERA

Ya despliega Natura su manto verde sobre todos los florecientes árboles, y extiende sus hojas de margaritas en los campos de hierba ya cubiertos; ya Febo saluda a los ríos límpidos, y se alegra del azul de los cielos;

mas, cansada, de nada se alegra ella, que firme continúa resistiendo.

Ya las alondras despiertan al alba con sus alas cubiertas de rocío; el halcón, en su elevado refugio, hace resonar el eco en el bosque; el tordo salvaje gorgojea notas, adormilado, quiere descansar:

en libertad y amor se regocijan,

sin temor a opresión o esclavitud. 
Now blooms the lily by the bank,

The primrose down the brae;

The hawthorn's budding in the glen,

And milk-white is the slae:

The meanest hind in fair Scotland

May rove their sweets amang;

But I, the Queen of a' Scotland,

Maun lie in prison strang.

I was the Queen o' bonie France,

Where happy I hae been;

Fu' lightly raise I in the morn,

As blythe lay down at e'en:

And I'm the sov'reign of Scotland,

And mony a traitor there;

Yet here I lie in foreign bands,

And never-ending care.

But as for thee, thou false woman,

My sister and my fae,

Grim Vengeance yet shall whet a sword

That thro' thy soul shall gae;

The weeping blood in woman's breast

Was never known to thee;

Nor th' balm that draps on wounds of woe

Frae woman's pitying e'e.

My son! my son! may kinder stars

Upon thy fortune shine;

And may those pleasures gild thy reign,

That ne'er wad blink on mine!

God keep thee frae thy mother's faes,

Or turn their hearts to thee:

And where thou meet'st thy mother's friend,

Remember him for me!

O! soon, to me, may Summer suns

Nae mair light up the morn!

Nae mair to me the Autumn winds

Wave o'er the yellow corn?

And, in the narrow house of death,

Let Winter round me rave;

And the next flow'rs that deck the Spring,

Bloom on my peaceful grave! 
Florece la azucena en la orilla, la primavera baja la ladera; el espino naciente en la cañada, y blanco como leche es el endrino: la altiva y hermosa cierva de Escocia puede vagabundear en la dulce Natura; pero yo, que soy la Reina de Escocia cautiva he de estar en esta prisión.

Yo era la Reina de la hermosa Francia, donde realmente feliz he sido;

suavemente me alzo en la mañana, para, confiada, llegar al final:

y soy la soberana de Escocia, donde abundaron mucho los traidores; aún yazco aquí en manos extrañas y bajo un gran cuidado interminable.

Mas para ti, tú, tan falsa mujer, hermana mía, también mi enemiga, cruel venganza cuando empuñe la espada que atravesando tu alma avanzará; la sangre que del pecho manará nunca antes la hubiste conocido; ni las heridas surgidas del miedo del compasivo ojo de una mujer.

¡Hijo mío! ¡Que la estrella mejor Ilumine siempre tu porvenir! ¡Y que todo placer colme tu reino pues durante el mío nunca brilló! Dios te guarde del sino de tu madre, o vuelvan sus corazones al tuyo: donde veas amigos de tu madre ¡que ya siempre lo recuerden por mí!

¡Oh! ¡Muy pronto los soles de verano no alumbrarán para mí la mañana! ¿Ni para mí los vientos del otoño silbarán sobre el dorado maíz? Y, allí, en la angosta casa de la muerte, dejad que el loco invierno me rodee; iy las flores que dé la primavera florecerán en mi tranquila tumba! 


\section{2. "To the Exiled Patriots"23, de Coleridge}

To The EXILEd Patriots

MARTYRS of FREEDOM-ye who firmly good

Stept forth the champions in her glorious cause,

Ye who against corruption nobly stood

For justice, liberty, and equal laws.

Ye who have urg'd the cause of man so well,

Whilst proud oppression's torrent swept along,

Ye who so firmly stood, so nobly fell,

Accept one ardent Briton's grateful song.

For shall oppression vainly think by fear

To quench the fearless energy of mind?

And glorying in your fall, exult it here

As tho' no honest heart were left behind?

Thinks the proud tyrant by the pliant law,

The timid jury and the judge unjust,

To strike the soul of liberty with awe

And scare the friends of freedom from their trust?

As easy might the Despot's empty pride

The onward course of rushing ocean stay ;

As easy might his jealous caution hide

From mortal eyes the orb of general day.

${ }^{23}$ Coleridge, Samuel Taylor, Essays on his own times, vol. 1, edited by William Pickering, 1850 pp. 19s. 
A LOS PATRIOTAS EN EL EXILIO

Mártires de libertad - vosotros que tan firmes

Avanzáis victoriosos en su gloriosa causa,

que nobles resistís contra la corrupción

por libertad, justicia y leyes de igualdad.

Vosotros que defendéis bien la causa del hombre,

mientras sigue avanzando la opresión cual torrente,

vosotros que aguantáis con firmeza y nobleza,

aceptad un cantar de gratitud británica.

¿Meditará opresión en vano por el miedo

para saciar la osada energía mental?

¿Y de vuestra caída aquí se jactará

como si no quedara un corazón honrado?

¿Piensa el tirano altivo con la flexible ley,

el tímido jurado y el poco justo juez,

golpear a la libertad en su alma con temor, y asustar al amigo fiel de la libertad?

Fácil puede el vacío orgullo de los déspotas

parar el tenaz curso del agitado océano;

su celosa prudencia fácil puede ocultar

a los ojos mortales la orbe del día usual. 
For like that general orb's eternal flame

Glows the mild force of virtue's constant light;

Tho' clouded by misfortune, still the same,

For ever constant, and for ever bright.

Not till eternal chaos shall that light

Before oppression's fury fade away ;

Not till the sun himself be lost in night;

Not till the frame of nature shall decay.

Go then secure, in steady virtue go,

Nor heed the peril of the stormy seas,

Nor heed the felon's name, the outcast's woe;

Contempt and pain, and sorrow and disease.

Tho' cankering cares corrode the sinking frame,

Tho' sickness rankle in the sallow breast;

Tho' death were quenching fast the vital flame,

Think but for what ye suffer, and be blest.

So shall your great examples fire each soul,

So in each free-born breast for ever dwell,

Till man shall rise above the unjust control,

Stand where ye stood, and triumph where ye fell. 
Como la llama eterna que tiene el usual orbe brilla con luz constante y suave la virtud; pese a la gris desgracia, como siempre se encuentra, para siempre constante, para siempre brillante.

No hasta que la luz brille del siempre eterno caos antes de que se apague la ira de la opresión; no hasta que el solar astro en la noche se pierda; no hasta que se corrompa el marco natural.

Id, por tanto, seguros buscando la virtud, no temáis al peligro de mares tempestuosos, no temáis al bandido, ni os asustéis del paria; desprecio y sufrimiento, pesar y enfermedad.

Pese a que la gangrena corroa lo que se hunde, y que la enfermedad duela en el pecho adusto; aunque la muerte apague vuestra llama vital, pensad por quién lucháis y seréis bendecidos.

Así, éste vuestro ejemplo avivará cada alma, y así vivirá siempre en cada pecho libre,

hasta que el hombre alcance el injusto control, estad donde yo estuve, triunfad donde caí. 


\section{3. "When I have borne in memory",24, de Wordsworth}

When I have borne in memory that has tamed Great Nations, how ennobling thoughts depart When men change Swords for Ledgers, and desert The Student's bower for gold, some fears unnamed I had, my Country !- am I to be blamed?

But when I think of Thee, and what Thou art, Verily, in the bottom of my heart,

Of those unfilial fears I am ashamed.

But dearly must we prize thee; we who find

In thee a bulwark for the cause of men;

And I by my affection was beguiled:

What wonder if a Poet now and then,

Among the many movements of his mind,

Felt for thee as a Lover or a Child!

${ }^{24}$ Compuesto en 1802. Para la presente traducción se ha utilizado: Wordsworth, William. The poetical works of William Wordsworth: with a memoir, vol. 3, Little, Brown and Co., 1865, pp.74s. 
Cuando recordar puedo que había amaestrado muchas Grandes Naciones, iqué ideas nobles surgieron cambiando el hombre espadas por cuentas, y dejando la fe del aprendiz por oro, miedo inédito tuve, ¡oh! ¡Mi País! - ¿Debo yo ser culpado?

Mas, cuando pienso en ti y en lo que representas, ciertamente, en el fondo de éste mi corazón, siento que me avergüenzo de esos miedos indignos. Mas debemos premiarte; nosotros que en ti hallamos un bastión que sostiene la causa de los hombres; y yo, que fui engañado por mis grandes afectos: ¡cuán grandioso sería si un Poeta hoy y antes, entre tantas ideas que en su mente germinan, pudiera a ti sentirte como un Amante o un Niño! 


\section{CONCLUSIÓN}

Con el presente trabajo hemos querido contribuir a la recuperación y la figura y la obra de Robert Burns, poco conocidas y traducidas en lengua española, poniendo de manifiesto su influencia en uno de los grupos de poetas más importantes de la historia de la literatura inglesa: los poetas 'lakistas'.

Podemos señalar que el reconocimiento y la admiración de los que goza Robert Burns en el mundo angloparlante se derivan tanto de la renovación de la lengua escocesa como de su exaltación de los derechos sociales y los valores nacionales escoceses. Así, los poetas 'lakistas', tras leer y estudiar su obra, no dudan en tomarlo como referencia y modelo para, en un principio, criticar su sociedad y alabar los valores en los que se desarrolla la Revolución Francesa, y finalmente mostrar su patriotismo frente a los intentos de dominación napoleónica.

REFERENCIAS BIBLIOGRÁFICAS

AMADO, Pedro, “Nelson, 'El héroe caído'”. Todo a babor. Historia Naval, 2009 [Fecha de consulta: 1 de octubre de 2009] http://www.todoababor.es/articulos/nel-alm.htm

Burke, Edmund, Reflections on the Revolution in France, 1790 [Fecha de consulta: 3 de septiembre de 2009] http://www.constitution.org/eb/rev fran.htm

BuRns, Robert. Poems, chiefly in the Scottish Dialect, Belfast: William Magee, 1793. , The works of Robert Burns: with an introduction and bibliography, Hertfordshire: Wordsworth Editions, 1994.

CALATRAVA EscoBaR, Juan. Estudios sobre la Revolución Francesa y el final del Antiguo Régimen. Tres Cantos: Akal, 1980.

CARLYE, Thomas, "Bums". Emerson, Ralph Waddo; Thomas Carlyle, Henry Swasey McKean, Charles Stearns Wheeler, Critical and miscellaneous essays, 1, James Munroe, 1838.

CoBBAN, Alfred, La interpretación social de la revolución francesa. Madrid: Narcea de Ediciones, 1971.

CODY, David, The French Revolution, 1987 [Fecha de consulta: 8 de septiembre de 2009] http://www.victorianweb.org/history/hist7.html

CunNingham, Allan, The Works of Robert Burns: with his life, 2 vols. London: James Cochrane and Co, 1834.

CURRIE, James, The Works of Robert Burns with an Account of his Life and a Criticism on his writings. To which are prefixed some observations on the character and condition of the Scottish peasantry. Nueva York: Lum. Borradaire,1826.

ELL, Paul, Act of Union - Virtual Library, Belfast:School of Sociology and Social Policy. The Queen's University of Belfast 2009 [Fecha de consulta: 7 de septiembre de 2009] http://www.actofunion.ie/

FOWLER, Richard Hindle. Robert Burns. London: Routledge, 1988. 
Hume, David, "Great Britain: Scots. Letter to Gilbert Elliot of Minto, July 1757". En Yapp, Peter. The Traveller's dictionary of quotation: who said what, about where? Kentucky: Routledge, 1983.

Low, Donald. Robert Burns: The Critical Heritage. London: Routledge, 1974.

MARTIN, Jean-Clément, La Révolution française: généalogie de l'ennemi, 2001 [Fecha de consulta: 15 de septiembre de 2009] http://www.cairn.info/article p.php?ID ARTICLE=RAI 0050069

MCCLURE, Dernick J., Scots and its Literature. Amsterdam: John Benjamin's Publishing, 1995.

MCGINTY, J. Walker. Robert Burns and Religion. Surrey: Ashgate Publishing, 2003.

McGUIRK, Carol, "Paradoxes of Self Expression and Tradition after 1740". En Robert Burns and the Sentimental Era. Atenas: University of Georgia, 1985, pp. 103-119.

PARLIAMENT HOUSE OF LORDS. Act of Union 1707, 2007 [Fecha de consulta: 11 de octubre de 2009] http://www.parliament.uk/actofunion/index.html

PHILP, MARK, Britain and the French Revolution, 2002. [Fecha de consulta: 4 de octubre de 2009] http://www.bbc.co.uk/history/british/

SoBoul, Albert, La Francia de Napoleón. Barcelona: Crítica, 1993.

SOUTHEY, Robert, The Life of Horatio Lord Nelson. Project Gutenberg, 1813 [Fecha de consulta: 9 de septiembre de 2009] http://infomotions.com/etexts/gutenberg/dirs/9/4/947/947.htm

SPECK, William Allen, Stability and strife (England, 1714-1760). Harvard U. P.,1978. A concise history of Britain 1707-1975. Cambridge University Press, 2003.

TAYLOR, Samuel, Robert Burns as a Poet and as a Man, 2 vols., New York: Baker and Scribner, 1848.

VovelLe, Michel, Introducción a la historia de la Revolución Francesa. Barcelona: Editorial Crítica, 2000.

WILKIE, George Scott,. Understanding Robert Burns. Verse, Explanation and Glossary, foreword by James Cosmo. Glasgow: Neil Wilson Publishing, 2009.

WHITNEY, Elizabeth, English Romanticism. University of Houston, 2000 [Fecha de consulta: 15 de septiembre de 2009] http://www.uh.edu/engines/romanticism/index.html

WoodS, Alan, "British poets and the French Revolution. Part Two: Wordsworth and Coleridge - The death of an ideal". En: In Defence of Marxism, 2003 [Fecha de consulta: 15 de septiembre de 2009] http://www.marxist.com/british-poets-french-revolution-2.htm

WORDSWORTH, William, "A Bard's Epitaph". En: Burns, Robert. The Complete Works of Robert Burns, edited by Alexander Smith. KayDreams,1855.

The Poetical Works of William Wordsworth: with a memoir, Little, Brown and Co., 1865.

. The Prelude. Bartleby, 2009 [Fecha de consulta: 15 de octubre de 2009] http://www.bartleby.com/145/ww295.html] 